Supporting Information

\title{
Title: Oxygen Bridged Hybrid Metallocene-Nonmetallocene Polymetallic Catalysts of Group 4 Metals for Bimodal Activity in Olefin Polymerization: Synthesis, Characterization, and Theoretical Investigation
}

\author{
Authors: Swadhin K. Mandal, ${ }^{\dagger}$ Prabhuodeyara M. Gurubasavaraj, ${ }^{\dagger}$ Herbert W. Roesky, ${ }^{\dagger *}$ \\ Gerald Schwab, ${ }^{\dagger}$ Dietmar Stalke, ${ }^{\dagger}$ Rainer B. Oswald, ${ }^{\S}$ and Volker Dolle ${ }^{\$}$ \\ ${ }^{\dagger}$ Institute of Inorganic Chemistry, University of Göttingen, Tammannstrasse 4, 37077 Göttingen \\ (Germany)

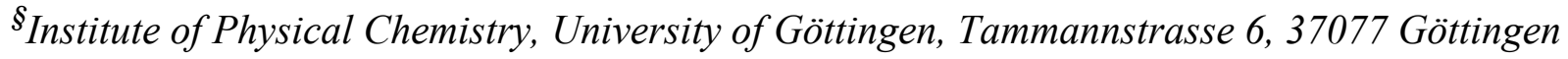 \\ (Germany) \\ ${ }^{\$}$ Basell R \& D Polymer Physics and Characterization, Industriepark, Hoechst, Frankfurt \\ (Germany) \\ * Author to whom correspondence should be addressed. E-mail: hroesky@gwdg.de
}




\section{Complete Reference (40):}

Frisch, M. J.; Trucks, G. W.; Schlegel, H. B.; Scuseria, G. E.; Robb, M. A.; Cheeseman, J. R.; Montgomery, J. A.; Vreven, Jr. T.; Kudin, K. N.; Burant, J. C.; Millam, J. M.; Iyengar, S. S.;

Tomasi, J.; Barone, V.; Mennucci, B.; Cossi, M.; Scalmani, G.; Rega, N.; Petersson, G. A.;

Nakatsuji, H.; Hada, M.; Ehara, M.; Toyota, K.; Fukuda, R.; Hasegawa, J.; Ishida, M.;

Nakajima, T.; Honda, Y.; Kitao, O.; Nakai, H.; Klene, M.; Li, X.; Knox, J. E.; Hratchian, H.

P.; Cross, J. B.; Bakken, V.; Adamo, C.; Jaramillo, J.; Gomperts, R.; Stratmann, R. E.;

Yazyev, O.; Austin, A. J.; Cammi, R.; Pomelli, C.; Ochterski, J. W.; Ayala, P. Y.; Morokuma,

K.; Voth, G. A.; Salvador, P.; Dannenberg, J. J.; Zakrzewski, V. G.; Dapprich, S.; Daniels,

A. D.; Strain, M. C.; Farkas, O.; Malick, D. K.; Rabuck, A. D.; Raghavachari, K.; Foresman, J. B.; Ortiz, J. V.; Cui, Q.; Baboul, A. G.; Clifford, S.; Cioslowski, J.; Stefanov, B. B.; Liu, G.; Liashenko, A.; Piskorz, P.; Komaromi, I.; Martin, R. L.; Fox, D. J.; Keith, T.; Al-Laham, M. A.; Peng, C. Y.; Nanayakkara, A.; Challacombe, M.; Gill, P. M. W.; Johnson, B.; Chen, W.; Wong, M. W.; Gonzalez, C.; Pople, J. A. Gaussian 03, Revision C.02, Gaussian, Inc., Wallingford CT, 2004. 
(a)

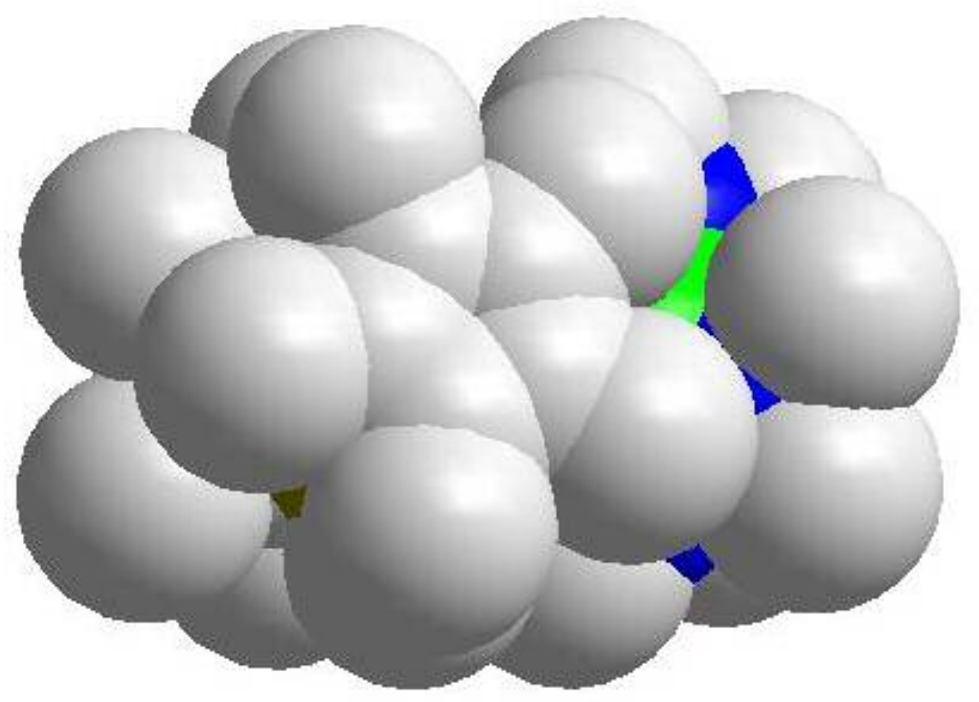

(b)

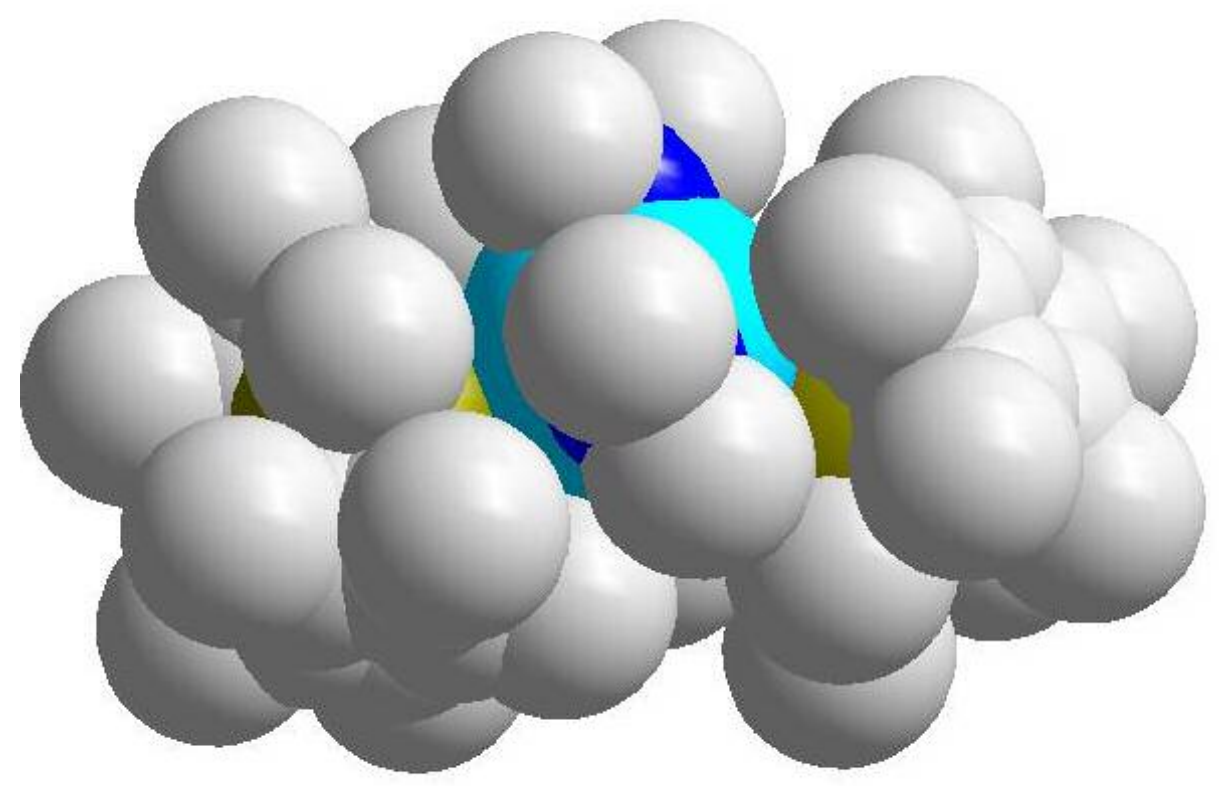

Figure S1. Space filling models of (a) 7 and (b) 9 revealing that in 7 the $\mathrm{Cp}^{*}$ units render more steric hindrance on the central metal ( $\mathrm{Ti}$ in bright green color) than that in $\mathbf{9}$ (Hf in sky blue color). 
Table S1. Results of Control Experiment on Polymerization Using Starting Precursors ${ }^{\mathrm{a}}$

\begin{tabular}{ccccc}
\hline catalyst & Monomer & $\begin{array}{c}\text { MAO: } \\
\text { catalyst }\end{array}$ & $\begin{array}{c}\text { Polymer } \\
(\mathrm{g})\end{array}$ & $\begin{array}{c}\mathrm{A} \\
\left(\times 10^{5}\right)\end{array}$ \\
\hline $\mathrm{Cp}_{2}{ }_{2} \mathrm{ZrMe}_{2}$ & ethylene & 400 & 0.680 & 1.36 \\
$\mathrm{Ti}\left(\mathrm{NMe}_{2}\right)_{4}$ & ethylene & 400 & 0.12 & 0.24 \\
$\mathrm{Hf}_{(}\left(\mathrm{NMe}_{2}\right)_{4}$ & ethylene & 400 & 0.06 & 0.12 \\
$\mathrm{Ti}\left(\mathrm{NMe}_{2}\right)_{4}$ & styrene & 800 & 0.39 & 0.39 \\
\hline
\end{tabular}

${ }^{a}$ Polymerization condition; $10 \mu \mathrm{mol}$ catalyst, $100 \mathrm{~mL}$ of toluene at $1 \mathrm{~atm}$ ethylene for $0.5 \mathrm{~h}$ or with $10 \mathrm{~mL}$ of styrene for $1 \mathrm{~h}$ at $25^{\circ} \mathrm{C}$. Activity (A) $=\mathrm{g}$ Polymer $/ \mathrm{mol}$ cat $\cdot \mathrm{h}$. 
Table S2. Calculated Atomic Coordinates for Neutral Compound 7

\begin{tabular}{lrrr}
$\mathrm{Zr}$ & 1.11463245 & 0.00472028 & -0.25069330 \\
$\mathrm{Ti}$ & -2.71058997 & -0.08547396 & 0.03189595 \\
$\mathrm{O}$ & -0.88501898 & -0.04525769 & 0.03050871 \\
$\mathrm{C}$ & 1.01466319 & -0.07805666 & -2.53273392 \\
$\mathrm{H}$ & 0.50286099 & -0.99464350 & -2.84757428 \\
$\mathrm{H}$ & 2.00349142 & -0.05177356 & -3.00912674 \\
$\mathrm{H}$ & 0.43491257 & 0.78022840 & -2.89607766 \\
$\mathrm{C}$ & 3.01970323 & -1.63413298 & 0.66854355 \\
$\mathrm{C}$ & 1.83269435 & -1.85054798 & 1.45673763 \\
$\mathrm{C}$ & 0.86273409 & -2.49387588 & 0.61720413 \\
$\mathrm{C}$ & 1.42321274 & -2.62485441 & -0.68885408 \\
$\mathrm{C}$ & 2.75374994 & -2.08168820 & -0.66324608 \\
$\mathrm{C}$ & 4.38868460 & -1.34202513 & 1.21972043 \\
$\mathrm{H}$ & 4.85369670 & -2.27679473 & 1.56768285 \\
$\mathrm{H}$ & 4.36710780 & -0.66226386 & 2.07572232 \\
$\mathrm{H}$ & 5.05646302 & -0.91441883 & 0.46828189 \\
$\mathrm{C}$ & 1.73184829 & -1.71162663 & 2.95413076 \\
$\mathrm{H}$ & 1.91281133 & -2.67678762 & 3.44979809 \\
$\mathrm{H}$ & 0.74189789 & -1.36821845 & 3.27552102 \\
$\mathrm{H}$ & 2.46659375 & -1.00483653 & 3.34807330 \\
$\mathrm{C}$ & -0.45143922 & -3.07068100 & 1.05928442 \\
$\mathrm{H}$ & -0.39385808 & -4.16793826 & 1.10708023 \\
$\mathrm{H}$ & -1.26973624 & -2.81146223 & 0.37889461 \\
$\mathrm{H}$ & -0.73160119 & -2.71775524 & 2.05531629 \\
$\mathrm{C}$ & 0.80776969 & -3.39961405 & -1.81916795 \\
$\mathrm{H}$ & 1.02583752 & -4.47260695 & -1.71297804 \\
$\mathrm{H}$ & 1.19394644 & -3.08706449 & -2.79353387 \\
$\mathrm{H}$ & -0.28087378 & -3.29183415 & -1.84118235 \\
$\mathrm{C}$ & 3.75134647 & -2.15594279 & -1.78745074 \\
$\mathrm{H}$ & 4.28955232 & -3.11520711 & -1.77751843 \\
$\mathrm{H}$ & 4.50470077 & -1.36341693 & -1.72042102 \\
$\mathrm{H}$ & 3.26794444 & -2.06605968 & -2.76520588 \\
$\mathrm{C}$ & 2.16199350 & 1.91948747 & 1.23821898 \\
$\mathrm{C}$ & 2.97598177 & 1.90910268 & 0.05083102 \\
$\mathrm{C}$ & 2.16063716 & 2.33339582 & -1.04577547 \\
$\mathrm{C}$ & 0.84355572 & 2.60467526 & -0.54494960 \\
$\mathrm{C}$ & 0.84929585 & 2.35887742 & 0.86484600 \\
$\mathrm{C}$ & 2.64911929 & 1.80407006 & 2.65788578 \\
$\mathrm{H}$ & 2.87898606 & 2.79941689 & 3.06618041 \\
$\mathrm{H}$ & 3.56180292 & 1.20763512 & 2.73845530 \\
$\mathrm{H}$ & 1.90004244 & 1.35433468 & 3.31794286 \\
$\mathrm{C}$ & 4.47600278 & 1.79444637 & -0.01113620 \\
& & & \\
\hline
\end{tabular}




$\begin{array}{lrrr}\mathrm{H} & 4.92499975 & 2.78627580 & -0.16672534 \\ \mathrm{H} & 4.81952710 & 1.15886602 & -0.83615997 \\ \mathrm{H} & 4.89531119 & 1.39350317 & 0.91329147 \\ \mathrm{C} & 2.67380119 & 2.65296923 & -2.42118473 \\ \mathrm{H} & 3.04127384 & 3.68884312 & -2.46382293 \\ \mathrm{H} & 1.89984056 & 2.55057501 & -3.18656903 \\ \mathrm{H} & 3.50641365 & 2.00166674 & -2.70808952 \\ \mathrm{C} & -0.30747439 & 3.17005797 & -1.32882518 \\ \mathrm{H} & -0.42885394 & 4.24432892 & -1.12743957 \\ \mathrm{H} & -1.25059505 & 2.67364217 & -1.07738073 \\ \mathrm{H} & -0.15163145 & 3.06004544 & -2.40654233 \\ \mathrm{C} & -0.27895998 & 2.65356264 & 1.81119172 \\ \mathrm{H} & -0.25072282 & 3.70404080 & 2.13738446 \\ \mathrm{H} & -0.23321642 & 2.03363003 & 2.71298970 \\ \mathrm{H} & -1.24944580 & 2.48108159 & 1.34093946 \\ \mathrm{~N} & -3.35321047 & -1.46430606 & -1.12701406 \\ \mathrm{C} & -2.71271212 & -1.74060128 & -2.41997845 \\ \mathrm{H} & -2.44033561 & -2.80492284 & -2.51210713 \\ \mathrm{H} & -1.80170977 & -1.14675874 & -2.53426316 \\ \mathrm{H} & -3.38663457 & -1.50192071 & -3.26075666 \\ \mathrm{C} & -4.55347617 & -2.28113725 & -0.91555772 \\ \mathrm{H} & -4.31191325 & -3.35708817 & -0.93645549 \\ \mathrm{H} & -5.30801220 & -2.09561672 & -1.69889731 \\ \mathrm{H} & -5.00475449 & -2.05754884 & 0.05377357 \\ \mathrm{~N} & -3.38387143 & -0.26631235 & 1.80740204 \\ \mathrm{C} & -4.82216729 & -0.15955847 & 2.09964798 \\ \mathrm{H} & -5.01696960 & 0.63235192 & 2.84166053 \\ \mathrm{H} & -5.22041542 & -1.10295388 & 2.50817834 \\ \mathrm{H} & -5.38084133 & 0.08639498 & 1.19191982 \\ \mathrm{C} & -2.58502636 & -0.50875941 & 3.01510713 \\ \mathrm{H} & -2.74334331 & 0.28684408 & 3.76247330 \\ \mathrm{H} & -1.52352816 & -0.53555358 & 2.76224538 \\ \mathrm{H} & -2.85412350 & -1.46706476 & 3.48965476 \\ \mathrm{~N} & -3.54041045 & 1.53255268 & -0.62870910 \\ \mathrm{C} & -3.92110316 & 1.76490566 & -2.02840490 \\ \mathrm{H} & -5.00358943 & 1.96190473 & -2.12114232 \\ \mathrm{H} & -3.68282967 & 0.89393426 & -2.64298240 \\ \mathrm{H} & -3.39252728 & 2.63600230 & -2.45320612 \\ \mathrm{H} & -3.87213130 & 2.68961520 & 0.21564743 \\ \mathrm{H} & -3.307598341 & 2.93896933 & 0.13898428 \\ \mathrm{H} & -3.64881383 & 2.47493226 & 1.26369786\end{array}$


Table S3. Calculated Atomic Coordinates for $\mathrm{Zr}$-centered Cation [ $\left.7^{+}(\mathrm{Zr})\right]$

$\begin{array}{lrrr}\mathrm{Zr} & 1.12080336 & 0.02086040 & 0.07206185 \\ \mathrm{Ti} & -2.74749241 & 0.00140715 & 0.02345726 \\ \mathrm{O} & -0.82874210 & 0.02405991 & 0.10939499 \\ \mathrm{C} & 2.87921784 & -1.76654806 & 0.75076890 \\ \mathrm{C} & 1.57453872 & -2.25655247 & 1.14846691 \\ \mathrm{C} & 0.82751635 & -2.55429880 & -0.03654599 \\ \mathrm{C} & 1.62677171 & -2.16486479 & -1.16559916 \\ \mathrm{C} & 2.91572524 & -1.73135477 & -0.68001270 \\ \mathrm{C} & 4.04893021 & -1.62375075 & 1.68800510 \\ \mathrm{H} & 4.43960723 & -2.61593481 & 1.95253280 \\ \mathrm{H} & 3.77991651 & -1.12975159 & 2.62765563 \\ \mathrm{H} & 4.87339979 & -1.06488473 & 1.24002827 \\ \mathrm{C} & 1.18294016 & -2.58281979 & 2.56632357 \\ \mathrm{H} & 1.58920332 & -3.56063039 & 2.85926523 \\ \mathrm{H} & 0.09898070 & -2.62885646 & 2.69691827 \\ \mathrm{H} & 1.57389735 & -1.85256945 & 3.28392159 \\ \mathrm{C} & -0.49999774 & -3.24938702 & -0.11984900 \\ \mathrm{H} & -0.43533693 & -4.10083598 & -0.80802183 \\ \mathrm{H} & -1.30405466 & -2.59378319 & -0.47584635 \\ \mathrm{H} & -0.79996118 & -3.64689838 & 0.85328399 \\ \mathrm{C} & 1.27632960 & -2.40802202 & -2.60998098 \\ \mathrm{H} & 1.60139419 & -3.41172561 & -2.91822021 \\ \mathrm{H} & 1.76615203 & -1.69574205 & -3.28224437 \\ \mathrm{H} & 0.19789719 & -2.35084965 & -2.78380667 \\ \mathrm{C} & 4.13259745 & -1.56867626 & -1.54890107 \\ \mathrm{H} & 4.47206892 & -2.55624248 & -1.89034728 \\ \mathrm{H} & 4.96524534 & -1.11070911 & -1.01325927 \\ \mathrm{H} & 3.93940818 & -0.97423330 & -2.44827073 \\ \mathrm{C} & 2.39987789 & 1.98998198 & 1.11766506 \\ \mathrm{C} & 3.12049367 & 1.75824151 & -0.09899045 \\ \mathrm{C} & 2.21740679 & 1.97202771 & -1.19731576 \\ \mathrm{C} & 0.96629542 & 2.46518638 & -0.65419256 \\ \mathrm{C} & 1.07730111 & 2.46840238 & 0.76647079 \\ \mathrm{C} & 2.98469988 & 2.02590073 & 2.50563084 \\ \mathrm{H} & 3.35082520 & 3.03543290 & 2.73870687 \\ \mathrm{H} & 3.83135007 & 1.34298653 & 2.61722246 \\ \mathrm{H} & 2.24474108 & 1.77448396 & 3.27340864 \\ \mathrm{H} & 4.60761013 & 1.58472157 & -0.20471524 \\ \mathrm{H} & 5.08980964 & 2.57030401 & -0.13669650 \\ \mathrm{H} & & & \\ \mathrm{H} & 2.92142593 & 0.97482623 & 0.60197705 \\ \mathrm{H} & 1.74860994 & 1.70845360 & -3.30370981 \\ \mathrm{H} & & 1.97435320 & -2.65727942 \\ \mathrm{H} & & & \\ \mathrm{H} & & & \\ \mathrm{H} & & & \end{array}$




$\begin{array}{lrrr}\mathrm{H} & 3.40877143 & 1.28080274 & -2.87388450 \\ \mathrm{C} & -0.19617866 & 2.97550998 & -1.45940578 \\ \mathrm{H} & -0.19925624 & 4.07357211 & -1.46921842 \\ \mathrm{H} & -1.15698393 & 2.63858739 & -1.05958521 \\ \mathrm{H} & -0.13971582 & 2.64800860 & -2.50208060 \\ \mathrm{C} & 0.07085861 & 2.99852818 & 1.74843934 \\ \mathrm{H} & 0.41470570 & 3.95486972 & 2.16479080 \\ \mathrm{H} & -0.09478437 & 2.32289566 & 2.59640436 \\ \mathrm{H} & -0.89347176 & 3.17503535 & 1.27184162 \\ \mathrm{~N} & -3.28991797 & -1.22500098 & -1.30962315 \\ \mathrm{C} & -2.71885764 & -1.07943846 & -2.66185212 \\ \mathrm{H} & -2.19507297 & -1.99699868 & -2.97279286 \\ \mathrm{H} & -2.00437413 & -0.24849206 & -2.69670743 \\ \mathrm{H} & -3.50483485 & -0.87774417 & -3.40489887 \\ \mathrm{C} & -4.26670642 & -2.32181782 & -1.22315640 \\ \mathrm{H} & -3.80622652 & -3.28568258 & -1.49228821 \\ \mathrm{H} & -5.10830248 & -2.15180926 & -1.91147694 \\ \mathrm{H} & -4.65999872 & -2.39387790 & -0.20807649 \\ \mathrm{~N} & -3.54791400 & -0.53247651 & 1.65169012 \\ \mathrm{C} & -4.79774710 & -0.02944544 & 2.24862725 \\ \mathrm{H} & -4.61741532 & 0.38902076 & 3.25071269 \\ \mathrm{H} & -5.53007048 & -0.84406708 & 2.35477745 \\ \mathrm{H} & -5.23759722 & 0.74389382 & 1.61784423 \\ \mathrm{C} & -2.93383958 & -1.59572457 & 2.47014019 \\ \mathrm{H} & -2.70755920 & -1.23148619 & 3.48388651 \\ \mathrm{H} & -2.00243514 & -1.94072603 & 2.01401499 \\ \mathrm{H} & -3.60870515 & -2.45964356 & 2.56587750 \\ \mathrm{~N} & -3.56745322 & 1.65294843 & -0.43630257 \\ \mathrm{C} & -4.35030765 & 1.99130187 & -1.63601089 \\ \mathrm{H} & -5.38425648 & 2.25529599 & -1.36548434 \\ \mathrm{H} & -4.38428417 & 1.14310557 & -2.32075495 \\ \mathrm{H} & -3.91552122 & 2.85377722 & -2.16529460 \\ \mathrm{C} & -3.54348009 & 2.76374596 & 0.53604020 \\ \mathrm{H} & -4.56716634 & 3.05875179 & 0.81212529 \\ \mathrm{H} & -3.04328716 & 3.65364841 & 0.12363961 \\ \mathrm{H} & -3.02512106 & 2.46732625 & 1.45345509\end{array}$


Table S4. Calculated Atomic Coordinates for Ti-centered Cation [ $\left.7^{+}(\mathrm{Ti})\right]$

$\begin{array}{lrrr}\mathrm{Zr} & -0.86778365 & -0.01114470 & -0.25956677 \\ \mathrm{Ti} & 2.80429197 & -0.01921210 & 0.02430351 \\ \mathrm{O} & 1.15850391 & -0.56309489 & 0.03445474 \\ \mathrm{C} & -0.71255361 & 0.05950243 & -2.52993435 \\ \mathrm{H} & 0.11581068 & 0.70676255 & -2.84178837 \\ \mathrm{H} & -1.63267933 & 0.45146839 & -2.98634979 \\ \mathrm{H} & -0.53762456 & -0.94199078 & -2.93871237 \\ \mathrm{C} & -1.90327052 & 2.05680610 & 1.02770462 \\ \mathrm{C} & -0.52613835 & 1.95433202 & 1.41671464 \\ \mathrm{C} & 0.27253827 & 2.28184405 & 0.26617480 \\ \mathrm{C} & -0.60210691 & 2.57575722 & -0.82739136 \\ \mathrm{C} & -1.94994582 & 2.39929198 & -0.36692174 \\ \mathrm{C} & -3.06468902 & 2.12256125 & 1.98064970 \\ \mathrm{H} & -3.20617319 & 3.16171400 & 2.31008556 \\ \mathrm{H} & -2.90311140 & 1.52237016 & 2.87852024 \\ \mathrm{H} & -4.00451895 & 1.80543552 & 1.52343657 \\ \mathrm{C} & -0.02679322 & 1.77474458 & 2.82612919 \\ \mathrm{H} & 0.00804921 & 2.73881411 & 3.35248425 \\ \mathrm{H} & 0.98295569 & 1.35207970 & 2.86363203 \\ \mathrm{H} & -0.67584025 & 1.11574261 & 3.41080749 \\ \mathrm{C} & 1.76564287 & 2.47942014 & 0.26163392 \\ \mathrm{H} & 2.04092725 & 3.53592965 & 0.38298826 \\ \mathrm{H} & 2.22589499 & 2.19644669 & -0.70572669 \\ \mathrm{H} & 2.24639039 & 1.98733514 & 1.12880598 \\ \mathrm{C} & -0.20373757 & 3.18597821 & -2.14000605 \\ \mathrm{H} & -0.22959083 & 4.28222662 & -2.06873655 \\ \mathrm{H} & -0.87814101 & 2.89817944 & -2.95072845 \\ \mathrm{H} & 0.81062322 & 2.90522294 & -2.44381311 \\ \mathrm{C} & -3.18446919 & 2.74246343 & -1.15554365 \\ \mathrm{H} & -3.36792264 & 3.82572767 & -1.13955635 \\ \mathrm{H} & -4.07667285 & 2.25977196 & -0.74804592 \\ \mathrm{H} & -3.09610861 & 2.44714529 & -2.20638384 \\ \mathrm{C} & -2.29989202 & -1.56231203 & 1.25615267 \\ \mathrm{C} & -3.10988031 & -1.28447837 & 0.09404375 \\ \mathrm{C} & -2.49142282 & -1.91486186 & -1.03847756 \\ \mathrm{C} & -1.28986899 & -2.55536381 & -0.58650947 \\ \mathrm{C} & -1.18039548 & -2.34749060 & 0.82944942 \\ \mathrm{C} & -2.69212609 & -1.34259943 & 2.69316373 \\ \mathrm{H} & -3.04660681 & -2.28497674 & 3.13252111 \\ \mathrm{H} & -3.50367883 & -0.61958178 & 2.79144473 \\ \mathrm{H} & -1.85833216 & -0.99756291 & 3.31520082 \\ \mathrm{C} & -4.50655341 & -0.72237119 & 0.08946170 \\ \mathrm{H} & -5.23979887 & -1.53919730 & 0.03901812 \\ \mathrm{H} & -4.69606019 & -0.07354095 & -0.77226729\end{array}$




$\begin{array}{lrrr}\mathrm{H} & -4.72689882 & -0.15238884 & 0.99443280 \\ \mathrm{C} & -3.12358172 & -2.05858501 & -2.39349542 \\ \mathrm{H} & -3.79242823 & -2.93027233 & -2.40632285 \\ \mathrm{H} & -2.38485551 & -2.20648355 & -3.18518750 \\ \mathrm{H} & -3.72625874 & -1.18541770 & -2.66188689 \\ \mathrm{C} & -0.37856983 & -3.42569143 & -1.40476919 \\ \mathrm{H} & -0.63619350 & -4.48577066 & -1.27506034 \\ \mathrm{H} & 0.66969906 & -3.30834567 & -1.10886938 \\ \mathrm{H} & -0.45377919 & -3.20623565 & -2.47363353 \\ \mathrm{C} & -0.15686444 & -2.98215777 & 1.72671171 \\ \mathrm{H} & -0.43705154 & -4.02001902 & 1.95453878 \\ \mathrm{H} & -0.07136248 & -2.45620274 & 2.68315413 \\ \mathrm{H} & 0.83335224 & -3.00519047 & 1.26150781 \\ \mathrm{~N} & 3.76807449 & -0.41060282 & 1.59073646 \\ \mathrm{C} & 5.23458108 & -0.32982389 & 1.76899611 \\ \mathrm{H} & 5.65174108 & -1.32751906 & 1.95902441 \\ \mathrm{H} & 5.47980631 & 0.31380945 & 2.62434512 \\ \mathrm{H} & 5.72199370 & 0.07899002 & 0.88189081 \\ \mathrm{C} & 3.10600033 & -0.94033321 & 2.80859429 \\ \mathrm{H} & 3.50003399 & -1.93689703 & 3.04887274 \\ \mathrm{H} & 2.02893787 & -1.02210503 & 2.65728669 \\ \mathrm{H} & 3.29923723 & -0.28088294 & 3.66546970 \\ \mathrm{~N} & 3.66372069 & -0.18196025 & -1.63394112 \\ \mathrm{C} & 3.03006827 & -0.78316635 & -2.83169442 \\ \mathrm{H} & 3.02034729 & -0.06485961 & -3.66201019 \\ \mathrm{H} & 2.00437676 & -1.08157009 & -2.61344886 \\ \mathrm{H} & 3.59593590 & -1.66887614 & -3.15022285 \\ \mathrm{C} & 5.06035058 & 0.23243236 & -1.89424505 \\ \mathrm{H} & 5.09833842 & 0.89877543 & -2.76602276 \\ \mathrm{H} & 5.69647487 & -0.63877152 & -2.09721842 \\ \mathrm{H} & 5.47915516 & 0.77647294 & -1.04223954\end{array}$

